\title{
Fluorescent Retinoic Acid Analogues as Probes for Biochemical and Intracellular Characterization of Retinoid Signaling Pathways
}

David R. Chisholm, ${ }^{\dagger}$ Charles W. E. Tomlinson, ${ }^{\dagger}{ }^{\dagger}$ Garr-Layy Zhou, ${ }^{\dagger}$ Claire Holden, ${ }^{\ddagger}$ Valerie Affleck, ${ }^{\S}$ Rebecca Lamb, ${ }^{\ddagger}$ Katherine Newling, ${ }^{\prime}$ Peter Ashton, " Roy Valentine, ${ }^{\perp}$ Christopher Redfern, ${ }^{\#}$

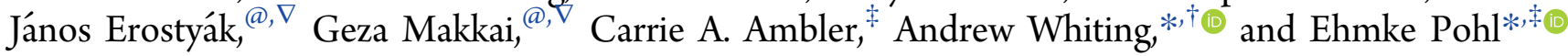

${ }^{\dagger}$ Department of Chemistry, Durham University, South Road, Durham DH1 3LE, U.K.

${ }^{*}$ Department of Biosciences, Durham University, South Road, Durham DH1 3LE, U.K.

${ }^{\S}$ LightOx Limited, Wynyard Park House, Wynyard Avenue, Wynyard, Billingham TS22 5TB, U.K.

"Genomics and Bioinformatics Laboratory, Department of Biology, University of York, York YO10 5DD, U.K.

${ }^{\perp}$ High Force Research Limited, Bowburn North Industrial Estate, Bowburn, Durham DH6 5PF, U.K.

"Northern Institute for Cancer Research, Medical School, Newcastle University, Newcastle upon Tyne NE2 4HH, U.K.

${ }^{\circledR}$ University of Pecs, Szentagothai Research Centre, Spectroscopy Research Group, Ifjusag u. 20, H-7624 Pecs, Hungary

${ }^{\nabla}$ University of Pecs, Faculty of Sciences, Ifjusag u. 6, H-7624 Pecs, Hungary

\section{Supporting Information}

ABSTRACT: Retinoids, such as all-trans-retinoic acid (ATRA), are endogenous signaling molecules derived from vitamin $A$ that influence a variety of cellular processes through mediation of transcription events in the cell nucleus. Because of these wideranging and powerful biological activities, retinoids have emerged as therapeutic candidates of enormous potential. However, their use has been limited, to date, due to a lack of understanding of the complex and intricate signaling pathways that they control. We have designed and synthesized a family of synthetic retinoids that exhibit strong, intrinsic, solvatochromatic fluorescence as multifunctional tools to interrogate these important biological activities. We utilized the unique photophysical characteristics of these fluorescent retinoids to develop

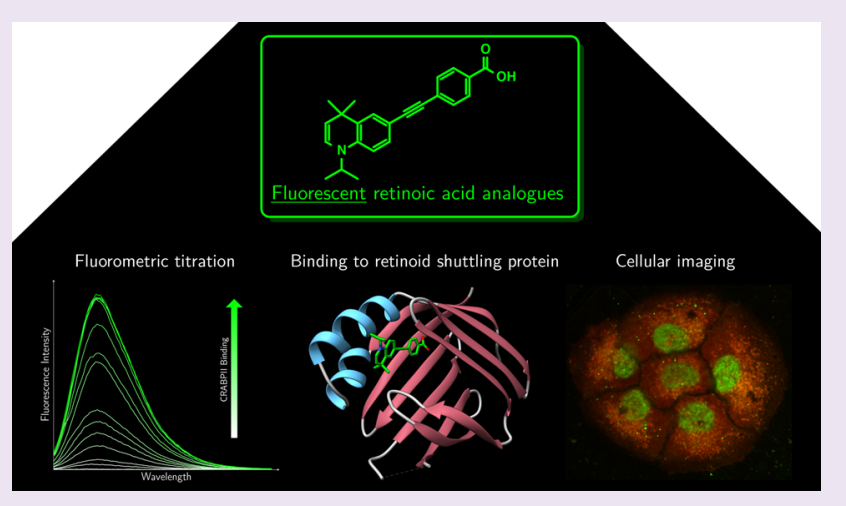
a novel in vitro fluorometric binding assay to characterize and quantify their binding to their cellular targets, including cellular retinoid binding protein II (CRABPII). The dihydroquinoline retinoid, DC360, exhibited particularly strong binding $\left(K_{d}=34.0 \pm 2.5 \mathrm{nM}\right)$, and we further used X-ray crystallography to determine the structure of the DC360-CRABPII complex to $1.8 \AA$, which showed that DC360 occupies the known hydrophobic retinoid binding pocket. Finally, we used confocal fluorescence microscopy to image the cellular behavior of the compounds in cultured human epithelial cells, highlighting a fascinating nuclear localization, and used RNA sequencing to confirm that the compounds regulate cellular processes similar to those of ATRA. We anticipate that the unique properties of these fluorescent retinoids can now be used to cast new light on the vital and highly complex retinoid signaling pathway.

$\mathrm{R}$ etinoids, such as all-trans-retinoic acid (ATRA) (Figure 1), make up a class of endogenous small molecules derived from or functionally related to vitamin A. ${ }^{1}$ These compounds exert a significant influence on a wide range of cellular processes in chordates, including differentiation, proliferation, apoptosis, and homeostasis, by regulating transcriptional control in the cell nucleus. ${ }^{2}$ To carry out this function, retinoids are high-affinity ligands for a family of nuclear receptor proteins known as the retinoic acid receptors (RARs) and retinoid X receptors (RXRs)..$^{3-5}$ Once they are bound to these receptors, dimerization allows the formation of nuclear receptor heterodimers (RAR/RXR), which function as stable platforms for interactions with short DNA sequences termed retinoic acid response elements (RAREs). ${ }^{6,7}$ These interactions facilitate transcriptional regulation of retinoic acid signaling pathways. ${ }^{8}$

As a result of the central role of retinoids in differentiation, endogenous retinoids have been used as potent therapeutics for the treatment of cancers and skin conditions $s^{2,3,9,10}$ and as key reagents for stem cell biology. ${ }^{11,12}$ However, despite the potential of retinoids for widespread therapeutic use, this potential has yet to be realized except in very limited

Received: October 12, 2018

Accepted: February 1, 2019

Published: February 1, 2019 

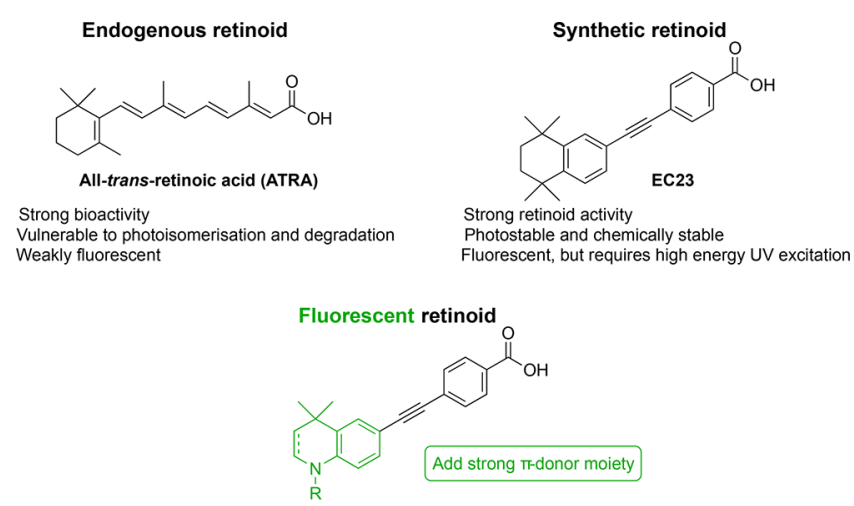

Strong retinoid activity

Highly fluorescent, excitation in UV to violet

Figure 1. Comparison of endogenous retinoid, ATRA, ${ }^{2,10}$ synthetic retinoid $\mathrm{EC} 23,{ }^{14}$ and design of a fluorescent retinoid.

contexts. ${ }^{3,10}$ The main reason for this is our lack of understanding of the temporal nature of retinoid signaling and the way in which the activation of different RAR types controls signaling pathways with respect to temporal- and cellspecific pathways of transcriptional regulation. Furthermore, it has become increasingly apparent that retinoids also act in a "nongenomic" manner, through the regulation of kinase phosphorylation and mRNA stability. ${ }^{2,13}$ These additional biochemical activities add further layers of complexity to retinoid signaling pathways.

Clearly, more work is needed to fully understand the diverse biological activities of these compounds and the signaling pathway(s) that they govern. However, ATRA and its endogenous isomers are highly susceptible to photoisomerization and degradation under typical laboratory lighting because of their conjugated polyene structure and exhibit poor chemical stability in general, meaning that the use of these compounds as routine reagents carries an inherent uncertainty and unreliability. ${ }^{14,15}$ Therefore, new compounds, probes, tools, and techniques are required to understand this complex biological pathway that has immense therapeutic potential.

In previous work, we developed a synthetic diphenylacetylene analogue of ATRA, known as EC23 (Figure 1),${ }^{14}$ which exhibits excellent photostability and biological activity comparable to that of ATRA in cellular differentiation assays. As a result, EC23 is now used as a potent and more reliable surrogate for ATRA in a number of laboratories. ${ }^{11,16-18}$ During development of EC23, we noticed that the compound exhibited fluorescence when excited with ultraviolet (UV) light and were intrigued by the possibility of conducting imaging studies to test the correlation between cellular localization and biological activity. However, because EC23 requires excitation at wavelengths $\left(\lambda_{\max }=300-310 \mathrm{~nm}\right)$ that are damaging to cells, attempting to image EC23 would not be possible without affecting the underlying cell biology. ${ }^{19}$ ATRA is also fluorescent under UV excitation; however, this is a much weaker effect, and excitation also initiates rapid photoisomerization, causing significant changes to the biological activity of the compound. ${ }^{14,20-22}$ Fluorescence imaging using these retinoids would, therefore, be unwise.

We anticipated that the incorporation of a strong $\pi$-donor moiety (e.g., $-\mathrm{NH}_{2}$ or $-\mathrm{NR}_{2}$ ) into the existing diphenylacetylene structure of EC23 would result in significant bathochromic shifts in absorption and emission, ideally allowing imaging using typical microscope excitation sources, e.g., 405 nm. ${ }^{23-29}$ Therefore, if the 1,1,4,4-tetramethyl-1,2,3,4-tetrahydronaphthalene (TTN) group of EC23 were substituted for an analogous, lipophilic, $\pi$-donating tetrahydroquinoline (THQ) or dihydroquinoline (DHQ) (Figure 1), then the resultant azaretinoid would exhibit improved fluorescence properties. This would enable cellular imaging and other biochemical and biophysical studies without external staining or fluorophore tagging and, most importantly, without affecting the underlying biology. In fact, examples of fluorescence imaging of bioactive compounds without the tagging of a fluorophore, which is likely to interfere with biological activity, are relatively rare in the literature. ${ }^{30}$

Therefore, we report herein the synthesis, photophysical properties, binding characteristics, cellular imaging, and initial biological characterization of a library of synthetic retinoids that exhibit strong, intrinsic, solvatochromatic fluorescence.

\section{RESULTS AND DISCUSSION}

Design and Synthesis. Starting with highly lipophilic, electron-rich tetrahydroquinolines (THQ) and dihydroquinolines $(\mathrm{DHQ}),{ }^{31,32}$ we designed a convergent synthesis strategy (Figure 2A) in which a range of these donor structures $(\mathbf{1 a}-\mathbf{f}$ ) were coupled using the Sonogashira or Suzuki-Miyaura methodology, either as the iodide or as the corresponding boronic ester, with a series of conjugated acceptors $(\mathbf{2 a}-\mathbf{e})$ to give retinoid esters $3 \mathbf{a}-\mathbf{i}$ (Figure 2B). The esters were then saponified using aqueous $\mathrm{NaOH}$ in tetrahydrofuran (THF) to give the desired retinoid structures (4a-i, DCXXX) (full synthesis routes and procedures are shown in the Supporting Information)..$^{33}$ The majority $(\mathbf{4 a}-\mathbf{g})$ were structurally related to EC23, a potent RAR agonist, ${ }^{16,34}$ but two other compounds, DC375 (4h) and DC479 (4i), were related to the RAR $\beta /$ RAR $\gamma$ agonist, TTNN. ${ }^{34}$ X-ray crystal structures of DC271 (4c) (previously reported as an RAR agonist ${ }^{35}$ ) and DC360 (4g) are shown in the Supporting Information, highlighting their planar structures.

In previous work, we found that synthetic retinoids that are "too long" or "too short", compared to ATRA, exhibit vastly reduced biological activities in TERA2.cl.SP12 human embryonal carcinoma stem cells. ${ }^{28}$ To ascertain if these observed differences were due to differences in cellular localization or poor binding affinity for the RARs, or both, we prepared fluorescent, biologically inactive retinoids by simply shortening or lengthening the acceptor structure. Longer retinoids, DC324 $4^{36}(4 \mathbf{j})$ and DC329 (4k), were prepared by attaching cinnamic (2e) and naphthoic acid (2c) acceptor structures, respectively, and a shorter retinoid, DC461 (41), was synthesized by addition of a simple benzoic acid (2f) moiety (Figure 2B).

Photophysical Characterization. Each compound exhibited two strong absorption bands in the UV and near-violet ranges due to the ability to access intramolecular charge transfer (ICT) excited states. ${ }^{23,24}$ As a result, the compounds exhibited solvatochromatic absorption characteristics (Figure 3 and Table S2), with a bathochromic shift in the absorbance band evident in nonpolar solvents (toluene) compared to polar solvents $[\mathrm{EtOH}$ and dimethyl sulfoxide (DMSO)]. Furthermore, the compounds also displayed highly solvatochromatic fluorescence emission due to efficient charge transfer from the donor THQ/DHQ structure to the acceptor carboxyl moiety, with a significant bathochromic shift observed in polar solvents (Figure 3 and Table S1). In comparison to EC23 (see Table 
A
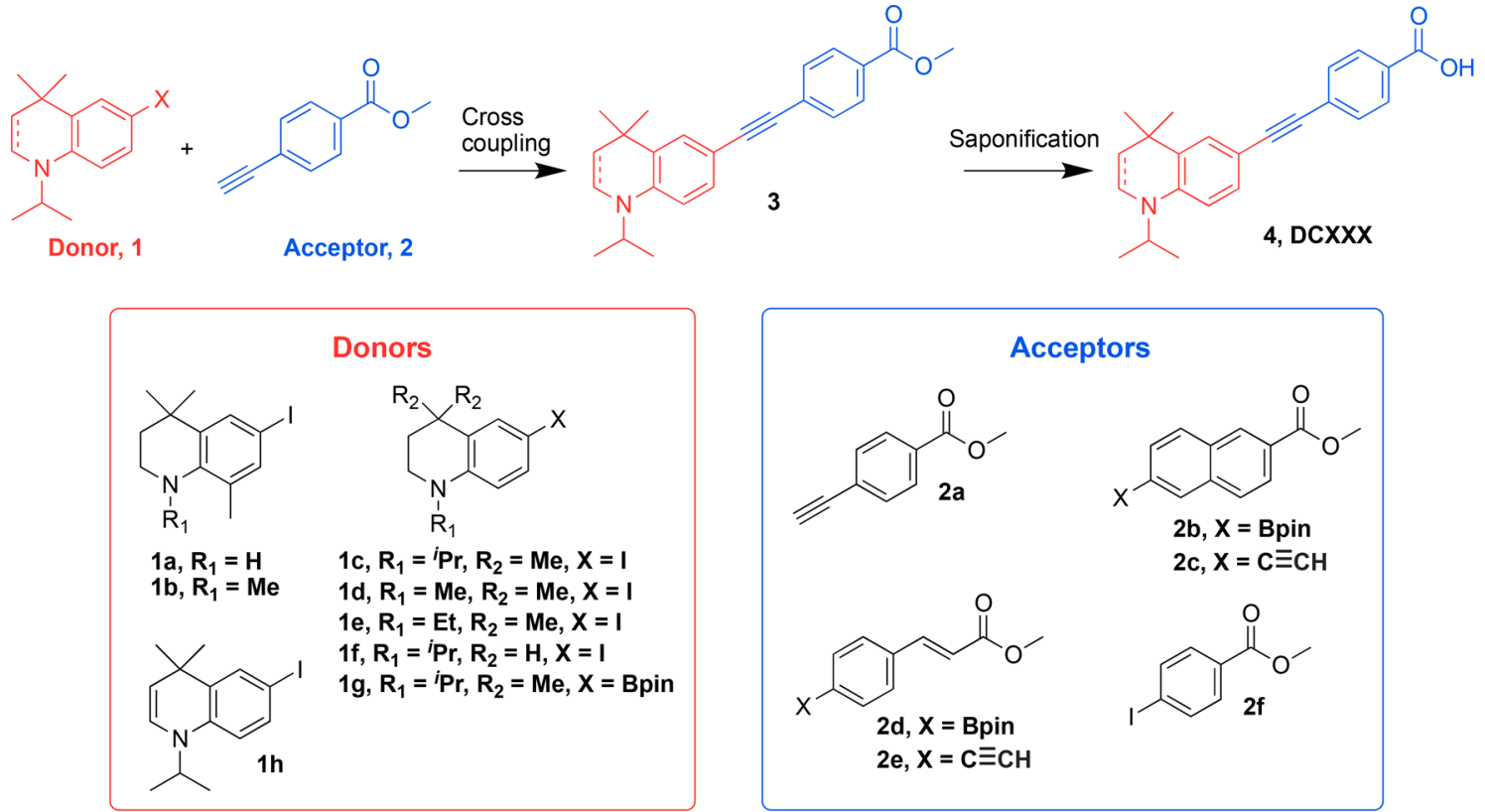

B

Analogues of all-trans-retinoic acid (ATRA)

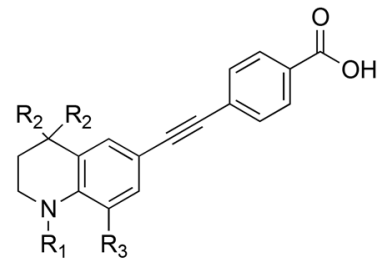

4a, $D C 122 \mathrm{R}_{1}=\mathrm{H}, \mathrm{R}_{2}=\mathrm{Me}, \mathrm{R}_{3}=\mathrm{Me}$ 4b, DC128 $R_{1}=M e, R_{2}=M e, R_{3}=M e$ 4c, DC271 $\mathrm{R}_{1}={ }^{i} \mathrm{Pr}, \mathrm{R}_{2}=\mathrm{Me}, \mathrm{R}_{3}=\mathrm{H}$ 4d, DC440 R $=M e, R_{2}=M e, R_{3}=H$ 4e, DC444 R $R_{1}=E t, R_{2}=M e, R_{3}=H$ 4f, DC476 $\mathrm{R}_{1}={ }^{i} \mathrm{Pr}, \mathrm{R}_{2}=\mathrm{H}, \mathrm{R}_{3}=\mathrm{H}$

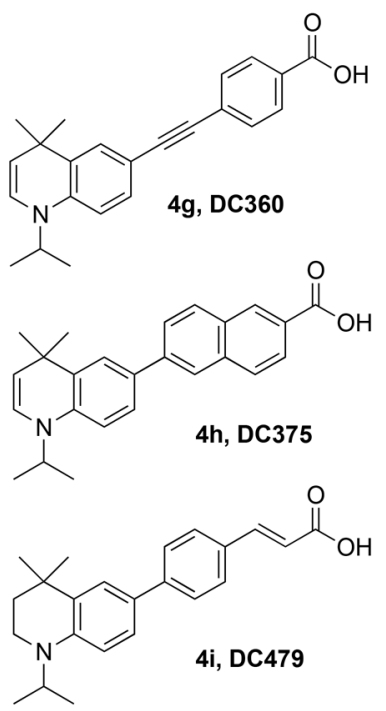

Lengthened/shortened compounds<smiles>CC(C)N1CCC(C)(C)c2cc(-c3ccc(C(=O)O)cc3)ccc2N1C(C)CCCOC(C)(C)C</smiles>

Figure 2. (A) Synthetic strategy for the assembly of a library of fluorescent retinoids involving cross coupling of donors $\mathbf{1}$ and acceptors $\mathbf{2}$ to provide esters 3. Subsequent saponification provided fluorescent retinoids 4. (B) Chemical structures of fluorescent retinoids, $4 a-i$, and lengthened and/or shortened compounds, $\mathbf{4} \mathbf{j}-\mathbf{l}$, designed to be non-retinoid in activity.

S1), each of the compounds exhibited significant bathochromic shifts in absorption and emission in all solvents, as originally anticipated. The longer compounds, DC $324^{36}(\mathbf{4 j})$ and DC329 (4k), exhibited a further bathochromic shift, by virtue of the increased charge separation between the donor and acceptor moiety. Equally, the shorter DC461 (41) exhibited a hypsochromic shift compared to the other compounds due to decreased charge separation.

It was noted that, for all of the compounds, the fluorescence emission in aqueous solutions was severely quenched (see Table S3), presumably due to aggregation effects caused by the high lipophilicity of the compounds. This was in stark contrast to the high quantum yields and hypsochromically shifted emission observed in toluene. We anticipated that this behavior would be particularly useful in cellular imaging and biophysical studies, by providing environmental information in addition to localization.

Retinoid Protein Binding. We next sought to assess the ability of the compounds to bind to a retinoid binding protein, and it was anticipated that the unique fluorescence properties of the fluorescent retinoids could be utilized to directly monitor binding to a ligand binding pocket. For these proof-ofprinciple experiments, we chose cellular retinoic acid binding protein II (CRABPII), a low-molecular weight (15.94 kDa), easily expressed, retinoid shuttling protein that is responsible for the translocation of retinoids from the cytoplasm to the nucleus and for transfer of retinoids to the RARs. ${ }^{37-39}$ 


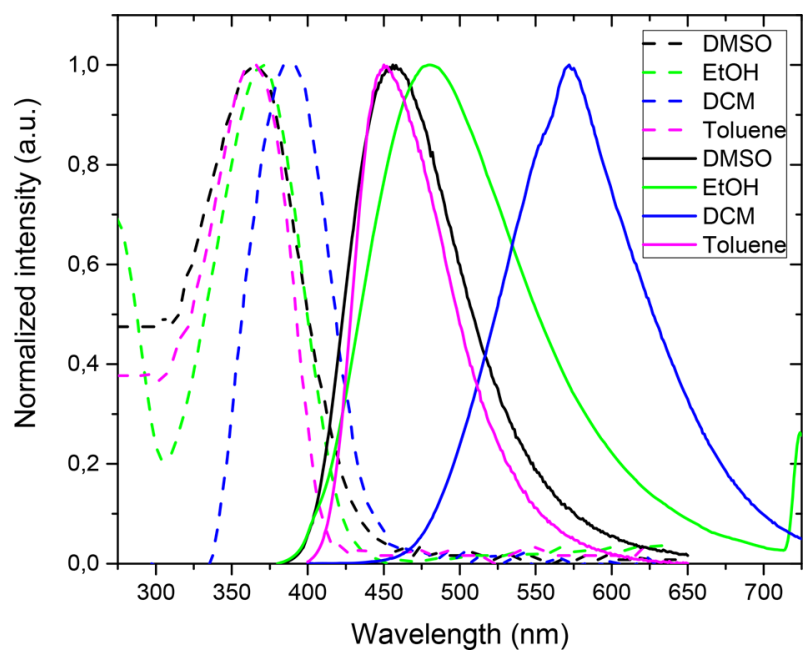

Figure 3. Normalized absorption (dashed) and emission (solid) spectra of DC360 $(4 \mathrm{~g})$ in a variety of solvents.

GST-CRABPII was produced via recombinant expression in Escherichia coli (BL21 DE3), before carrying out purification based on affinity for glutathione-sepharose. ${ }^{37}$ The purity was assessed by sodium dodecyl sulfate-polyacrylamide gel electrophoresis and confirmed by mass spectrometry before the determination of concentration by absorption measurements at $280 \mathrm{~nm}$. We then conducted an initial fluorometric binding study using DC360 (4g). A titration approach was used in which CRABPII was gradually titrated into a phosphate-buffered saline solution of the retinoid (50 nM) in a quartz cuvette, and the fluorescence signal was then measured after the system had equilibrated. As Figure 4A shows, at $0 \mathrm{nM}$ CRABPII, the fluorescence signal from the compound was heavily quenched and not detectable. However, as CRABPII was added, a significant increase in fluorescence intensity was observed. At higher concentrations ([CRABPII] $>100 \mathrm{nM}$ ), the increase in fluorescence intensity plateaued, and no further increase was evident. The observed emission wavelength in this experiment was similar to that in toluene, suggesting that the compound was localized in a nonpolar environment. $^{40,41}$ Furthermore, the observation that the increase in emission intensity was particularly large and that this increase plateaued suggested that DC360 (4g) binds to the insulated, hydrophobic retinoid binding site of CRABPII, where it is no longer quenched by neighboring molecules, and exhibits a saturable binding.

To determine the strength of this binding interaction, we conducted the fluorometric titration experiment in duplicate and recorded the emission intensity at $460 \mathrm{~nm}$ with excitation of DC360 (4g) at $340 \mathrm{~nm}$ as the CRABPII concentration was increased. The values of emission intensity at $460 \mathrm{~nm}$ were then processed, taking dilution and the minor contribution in fluorescence signal from CRABPII and the solvent into account. The processed emission intensity values were then plotted against the CRABPII concentration to give a binding isotherm (Figure 4B).

To quantify the strength of interaction, nonlinear regression analysis was performed to determine the dissociation binding constant, $K_{\mathrm{d}}$ of the interaction between DC360 (4g) and CRABPII. We employed the Hill equation, ${ }^{42}$ using GraphPad Prism 7.0d, and determined the $K_{\mathrm{d}}$ of the interaction between DC360 and CRABPII to be $34.0 \pm 2.5 \mathrm{nM}$, a value similar to that determined in the literature for ATRA $\left(K_{d}=14.2 \mathrm{nM}\right){ }^{37}$ This confirmed that DC360 (4g) bound to CRABPII with a high affinity and indicated that the hypsochromically shifted emission wavelength and high intensity were due to the compound binding to the insulated retinoid binding pocket.

The binding affinities of the other fluorescent retinoids were then assessed using the same approach. As Table 1 shows, all of those compounds designed to be active retinoids $(4 \mathbf{a}-\mathbf{i})$ were excellent CRABPII ligands with submicromolar binding affinities. It was further clear that the different $R_{1}-R_{3}$ substituents caused appreciable changes in the binding affinities for CRABPII. First, those compounds with $\mathrm{R}_{1}={ }^{i} \mathrm{Pr}$ (e.g., $4 c$ and $4 g$ ) exhibited $K_{d}$ values lower than the values of those with smaller $R_{1}$ groups (4d and $4 \mathbf{e}$ ). Second, the increased rigidity of the DHQ of DC360 (4g) appears to improve the binding affinity compared to that of the corresponding THQ compound, DC271 (4c). Third, when the geminal dimethyl group $\left(R_{2}\right)$ was removed (DC476, $\mathbf{4 g}$ ), a decrease in binding affinity was observed, indicating that this group is a desirable structural motif for binding to CRABPII. Finally, when $\mathrm{R}_{3}=\mathrm{Me}(\mathbf{4} \mathrm{a}$ and $\mathbf{4 b})$, a significant improvement in binding affinity was observed over the corresponding $\mathrm{R}_{3}=\mathrm{H}$ compound, DC440 (4d), suggesting that this ortho methyl group is also a desirable structural motif. ${ }^{31}$ The two compounds derived from known retinoid TTNN (DC375,

\section{A}

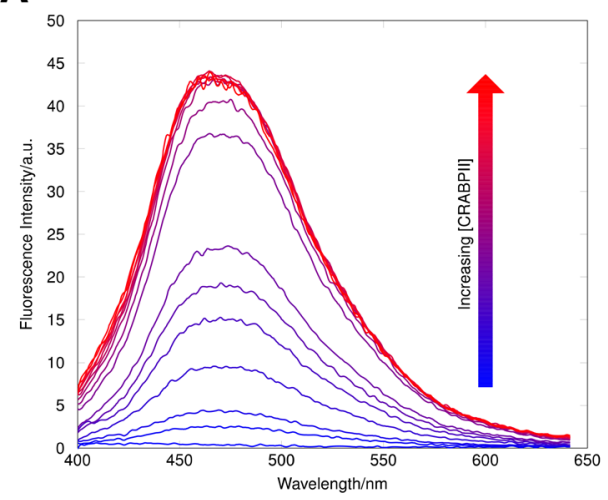

B

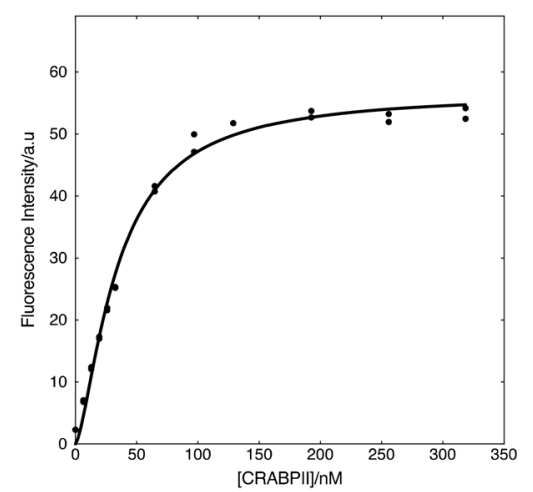

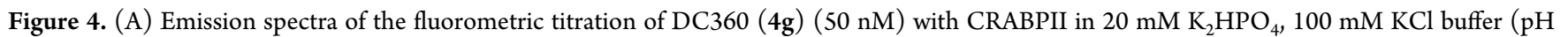
7.4). $\lambda_{\mathrm{ex}}=340 \mathrm{~nm}$. [CRABPII] $=0-350 \mathrm{nM}$. (B) Fluorometric titration of DC360 (4g) $(50 \mathrm{nM})$ with CRABPII in $20 \mathrm{mM} \mathrm{K}_{2} \mathrm{HPO}_{4}, 100 \mathrm{mM} \mathrm{KCl}^{-}$ buffer $(\mathrm{pH} 7.4) \cdot \lambda_{\mathrm{ex}}=340 \mathrm{~nm}$, and $\lambda_{\mathrm{em}}=460 \mathrm{~nm}$. 
Table 1. Dissociation Constants, $K_{\mathrm{d}}$, Determined Using the Hill Equation from Analysis of the Fluorometric Titration of the Fluorescent Retinoids with CRABPII

$\begin{array}{cc}\text { fluorescent retinoid } & K_{\mathrm{d}}(\mathrm{nM}) \pm \text { standard error } \\ \text { DC122 }(\mathbf{4 a}) & 107.3 \pm 5.9 \\ \text { DC128 }(\mathbf{4 b}) & 68.3 \pm 1.8 \\ \text { DC271 }(\mathbf{4 c}) & 49.1 \pm 2.6 \\ \text { DC440 (4d) } & 124.8 \pm 4.3 \\ \text { DC444 (4e) } & 94.0 \pm 3.7 \\ \text { DC476 (4f) } & 88.3 \pm 2.1 \\ \text { DC360 (4g) } & 34.0 \pm 2.5 \\ \text { DC375 (4h) } & 62.5 \pm 2.6 \\ \text { DC479 (4i) } & 51.6 \pm 3.2 \\ \text { DC324 }(\mathbf{4 j}) & \geq 875.0 \pm 118.4^{a} \\ \text { DC329 (4k) } & 132.0 \pm 10.1 \\ \text { DC461 }(\mathbf{4 l}) & 385.4 \pm 28.5\end{array}$

${ }^{a}$ The fluorescence signal could not be saturated under the experimental conditions $([\mathrm{CRABPII}]=0-1500 \mathrm{nM})$.

4h, and DC479, 4i) exhibited similarly strong binding when compared to that of the corresponding diphenylacetylenes DC271 (4c) and DC360 (4g).

When the fluorometric binding titrations were conducted with longer retinoids, DC324 (4j) and DC329 (4k), higher concentrations of CRABPII were required to saturate the binding signal. Furthermore, the intensity of the emission from both was much reduced and the wavelength longer compared to those of the other fluorescent retinoids (see Figures 14 and 15). These observations first of all showed that both compounds exhibit reduced binding affinity for CRABPII but, moreover, indicated that both the intensity and wavelength of the emission of these compounds can be used to infer environmental information. Indeed, the weaker and longer wavelength emission suggests that the compounds are localized in a more exposed, polar environment rather than the insulated retinoid binding site of CRABPII. Rather than exhibiting specific binding, the compounds may be binding nonspecifically and are therefore simply solvated by CRABPII.

DC461 (4l) also required significantly higher concentrations of CRABPII to reach saturation according to the fluorescence binding signal. However, in contrast to the longer compounds, DC461 exhibited the shorter wavelength, intense, emission
$(430 \mathrm{~nm})$ more akin to the those of other fluorescent retinoids (see Figure S16). This observation indicated that DC461 (41) is capable of entering the insulated CRABPII binding pocket but exhibits much weaker binding affinity, perhaps due to clashes between the entrance of the pocket (Phe16 and Val77) and the THQ moiety. ${ }^{40}$ Thus, the design strategy to deliberately synthesize shorter or longer fluorescent retinoids was successful on the basis of the reduced binding affinity for CRABPII.

To confirm that the modulation of the fluorescence properties of the fluorescent retinoids upon addition of CRABPII was due to specific binding, i.e., to the retinoid binding site, and not simply due to solvation, we crystallized DC360 (4g) with CRABPII. Crystallization was achieved using purified CRABPII, incubated with a molar excess of DC360, as a protein solution in a hanging drop experiment. Crystals were then cryoprotected, frozen in liquid nitrogen, and dry-shipped to the Diamond Light Source (beamline I02) for diffraction experiments. The resulting data were processed using the CCP4 suite of programs and solved using molecular replacement from a literature structure (Protein Data Bank entry 2FR3; further experimental detail given in the Supporting Information). ${ }^{43}$ The unbiased $F_{o}-F_{c}$ electron density map clearly showed DC360 (4g) occupying the retinoid binding site, and the structure (Figure $5 \mathrm{~A}$ ) was then refined to $1.80 \AA$ $\left(R=0.17\right.$, and $\left.R_{\text {free }}=0.24\right)$.

The compound is anchored inside the pocket (Figure 5B) via polar interactions between the carboxylate and Arg133/ Tyr135 and by a bifurcated hydrogen bond with $\operatorname{Arg} 112$ via a conserved water molecule $\left(\mathrm{H}_{2} \mathrm{O}-12\right)$ evident in other retinoid complexes in the literature. ${ }^{41}$ The DHQ hydrophobic region forms extensive van der Waals interactions with Leu29 and Ala33 among other residues on $\mathrm{H} 1$ and Val25 and Leu20 on $\mathrm{H} 2$, while Phe16 on $\mathrm{H} 1$ forms an offset $\mathrm{T}$-shaped $\pi-\pi$ interaction with the benzoate phenyl moiety. Furthermore, DC360 (4g) was found to exhibit a binding mode nearly identical to that of ATRA, ${ }^{40,41}$ confirming that it can be characterized as a retinoid. The crystal structure of the DC360-CRABPII complex also confirmed that the short wavelength, high-intensity emission of the fluorescent retinoids observed in the fluorometric titration was due to specific, highaffinity binding to CRABPII. In light of this, we predicted that
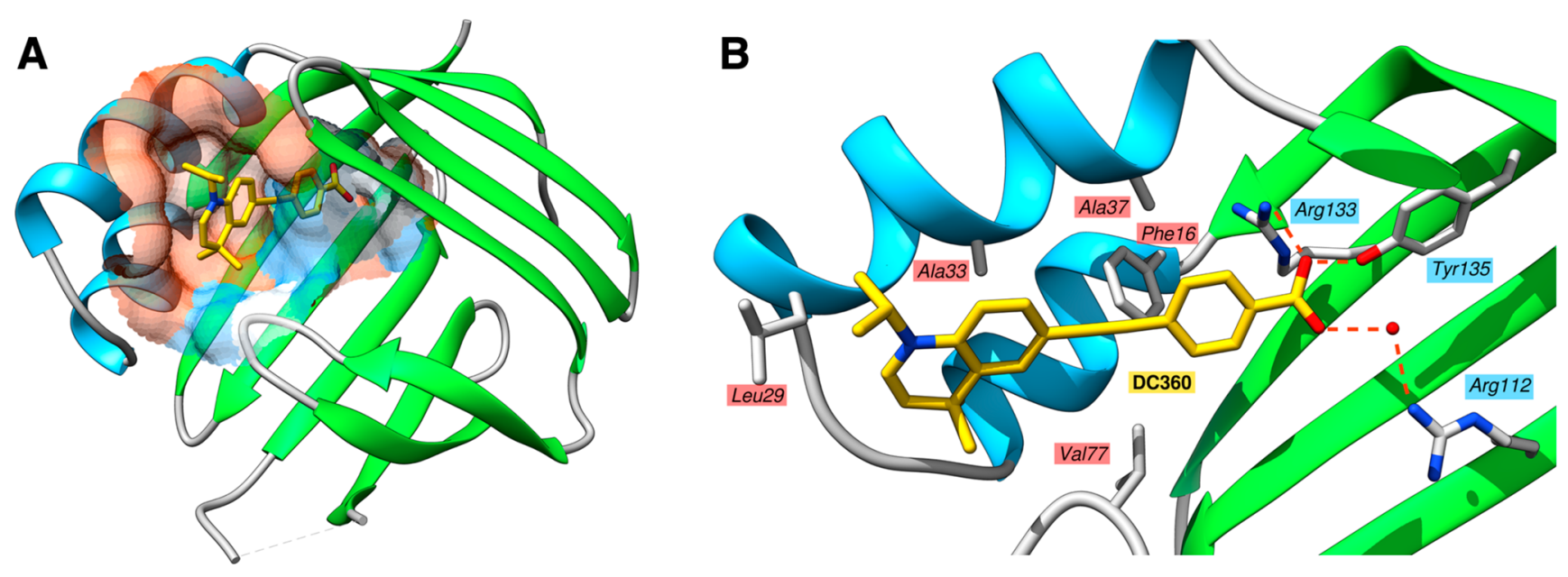

Figure 5. (A) CRABPII-DC360 complex with the hydrophobicity surface of the retinoid binding pocket highlighted. ${ }^{44}$ (B) Key interactions between DC360 (4g) and the CRABPII binding pocket. 


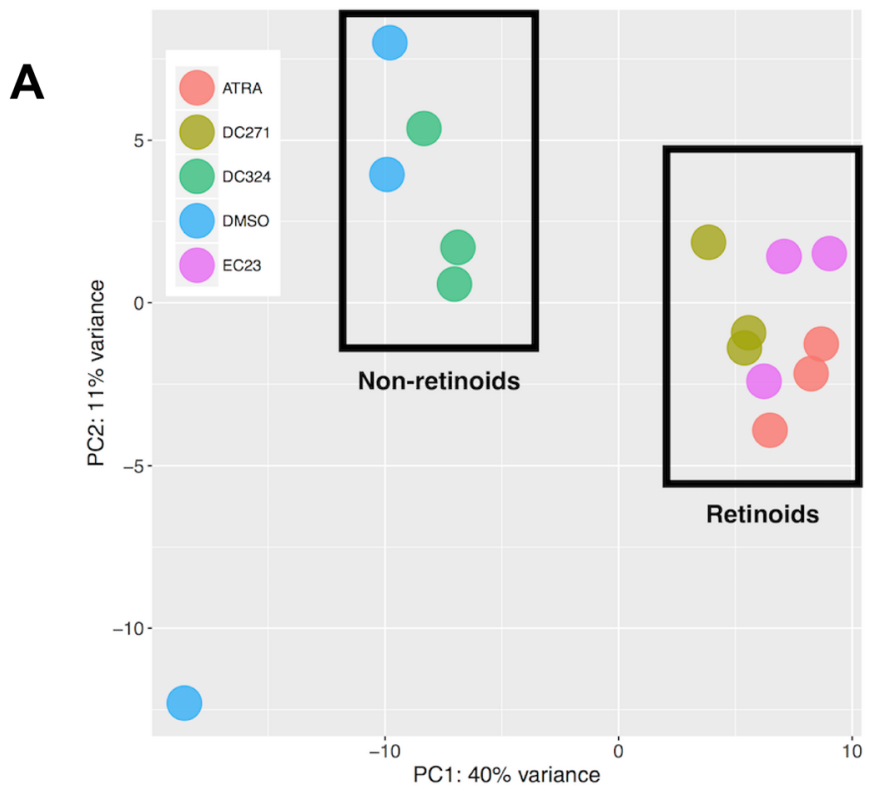

B Gene Ontology (GO) Gene sets:

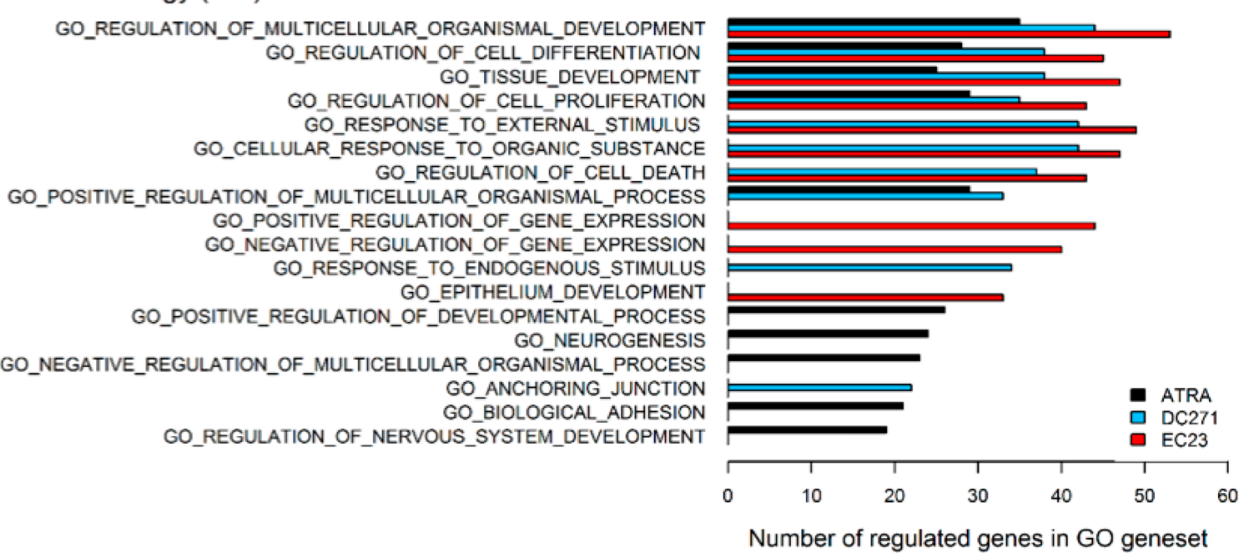

Figure 6. (A) Principle component analysis of RNA sequencing data collected from human epithelial cells treated with ATRA, EC23, or fluorescent retinoid DC271 (4c). (B) Gene ontology (GO) analysis of the number of genes regulated within a regulatory pathway for ATRA, EC23, and DC271 (4c) represented as a grouped horizontal bar plot (ATRA, DC271, and EC23 as per the legend), with GO regulatory pathways as group labels.

these compounds were suitable to be shuttled to the cell nucleus via CRABPII in a cellular context and, therefore, engage in RAR-mediated gene modulation.

Biological Characterization and Cellular Imaging. Having established that the majority of the fluorescent retinoids were excellent ligands for CRABPII, we wanted to compare the impact of retinoid treatment on gene regulation in human epithelial cells. To assess this, RNA sequencing was performed on cells treated with either active retinoids, ATRA, EC23, and DC271 (4c) or the "overly long" DC324 (4j).

Principal component analysis (PCA) (Figure 6A) showed that cells treated with EC23 or fluorescent retinoid DC271 (4c) differentially regulated $38-44 \%$ of the genes differentially expressed in response to ATRA. Importantly, retinoid-treated cell populations were completely distinct from those cells treated with either DC324 $(\mathbf{4 j})$ or DMSO. These data suggest that DC271 (4c) can be regarded as a retinoid, able to elicit cellular responses consistent with those of endogenous retinoid ATRA and synthetic retinoid EC23. ${ }^{14,17}$ Furthermore, PCA showed that DC324 (4j) can be regarded as biologically inactive as a retinoid and that the poor binding affinity for
CRABPII as determined by the in vitro fluorometric binding titration can be related to downstream biological activity (or lack thereof).

Figure $6 \mathrm{~B}$ also summarizes a gene ontology analysis [GO analysis (see the Supporting Information)] that suggests that EC23 and DC271 (4c) are better than ATRA at regulating cell differentiation and related processes, likely to be a result of the greater stability in vitro of the synthetic retinoids compared to that of ATRA. ${ }^{11,14,45}$ Conversely, protective responses to external agents tend to be upregulated by the synthetic retinoids but not ATRA, and ATRA, surprisingly for epithelial cells, upregulates neurogenesis genes in contrast to EC23 or DC271 (4c) (see Figure S29). This exemplifies the complexities involved in the signaling pathways governed by retinoids and underlines the need for an improved understanding of these fascinating behaviors.

The ability to image the localization of a fluorescent retinoid in human keratinocyte cells ( $\mathrm{HaCaT})$ was examined using confocal microscopy. HaCaTs were treated with $1 \mu \mathrm{M} \mathrm{DC271}$ (4c) and then imaged after incubation for $72 \mathrm{~h}$, a time period that has been shown to be required for the regulation of key 
differential genes in response to treatment with ATRA and 9cis-retinoic acid in these cells. ${ }^{46}$ The fluorescence emission from the compound could be detected at around 450-480 nm when DC271 (4c) was excited with 365-405 nm light (Figure 7). The distribution of the fluorescence signal varied somewhat

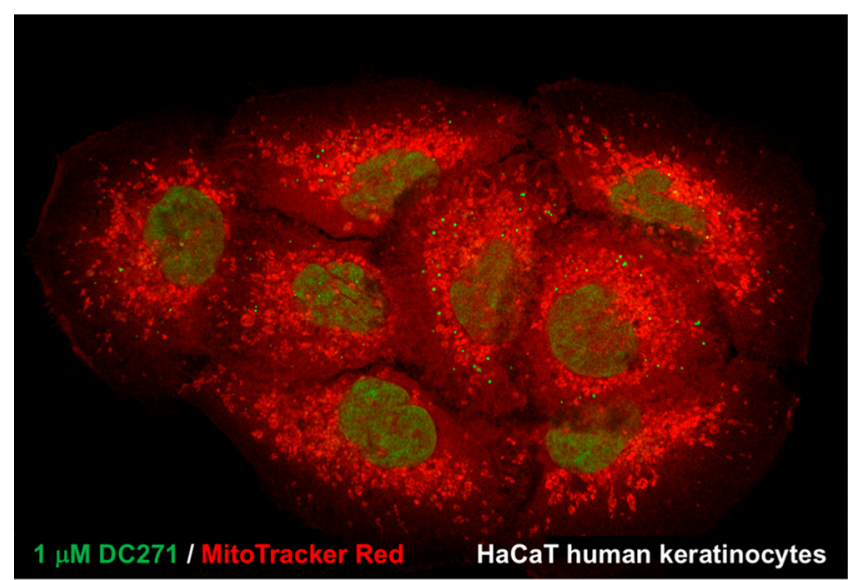

Figure 7. $\mathrm{HaCaT}$ cells treated with $1 \mu \mathrm{M} \mathrm{DC} 271$ and MitoTracker Red and imaged using a Zeiss 880 confocal microscope with $405 \mathrm{~nm}$ and $594 \mathrm{~nm}$ lasers.

depending on compound concentration and the duration of treatment; however, in general, the compound could be detected diffusely in the nucleus and in some brightly fluorescent, punctate structures in the cytoplasm (presumably lipid vesicles). Given the highly lipophilic structure of DC271 (4c), we were concerned that the compound might localize nonspecifically in nonpolar, membrane-rich environments such as mitochondria. However, this was not the case, as we detected minimal co-labeling of DC271 with the mitochondrion-specific dye MitoTracker Red. The nuclear localization suggests that DC271 (4c) is capable of engaging with the retinoid protein machinery (including CRABPII) required to translocate the endogenous retinoid, ATRA, into the nucleus, the suspected site of RAR-mediated gene regulation. ${ }^{1}$

Conclusions. In summary, we have demonstrated the design, synthesis, and characterization of a family of synthetic retinoids that exhibit strong fluorescence. The solvatochromatic behavior of the compounds has been applied in the development of a novel fluorometric titration assay that quantified the strength of binding to a retinoid binding protein involved in retinoid signaling, CRABPII, and could be applied to any other retinoid binding protein. These experiments confirmed not only that the majority of the fluorescent retinoids are excellent ligands for this important carrier protein but also that this strong binding affinity can be significantly diminished by increasing or decreasing the length of the compound. We further showed that the observed modulation of the fluorescence behavior by CRABPII was due to specific binding to the retinoid binding site by determining the crystal structure of CRABPII in complex with DC360 (4g). The fluorescent retinoids were then applied to a range of cellular imaging experiments that demonstrated that DC271 (4c) can be easily imaged in a cellular context, that changes in localization can be monitored over time, and that the compounds can be localized to the cell nucleus, the site of RAR-mediated gene regulation. Finally, we showed that DC271 (4c) activates the transcription of the same genes activated by ATRA and EC23 and that a longer fluorescent compound, DC324 (4j), is an effective non-retinoid, fluorescent control compound. We now look forward to applying this multifunctional family of fluorescent retinoids toward understanding the complex intricacies of retinoid biological activity, in the hope that the unravelling of these behaviors can lead to unlocking the immense therapeutic potential of both endogenous and synthetic retinoids.

\section{ASSOCIATED CONTENT}

\section{Supporting Information}

The Supporting Information is available free of charge on the ACS Publications website at DOI: 10.1021/acschembio.8b00916.

Detailed synthetic procedures; ${ }^{1} \mathrm{H},{ }^{11} \mathrm{~B}$, and ${ }^{13} \mathrm{C}$ NMR spectra; photophysical characterization data; fluorometric binding assay data; small molecule and protein crystallography data; and biological methods (PDF)

\section{AUTHOR INFORMATION \\ Corresponding Authors \\ *E-mail: ehmke.pohl@durham.ac.uk. \\ *E-mail: andy.whiting@durham.ac.uk.}

ORCID ${ }^{\circ}$

Christopher Redfern: 0000-0002-1833-8048

Andrew Whiting: 0000-0001-8937-8445

Ehmke Pohl: 0000-0002-9949-4471

\section{Notes}

The authors declare no competing financial interest.

\section{ACKNOWLEDGMENTS}

D.R.C. thanks the EPSRC, BBSRC, and High Force Research Ltd. for doctoral funding. C.W.E.T. thanks the MRC (MR/ N0186481/1) and High Force Research Ltd. for doctoral funding. G.-L.Z. thanks the EPSRC and High Force Research Ltd. for doctoral funding.

\section{REFERENCES}

(1) Chambon, P. A. (1996) Decade of Molecular Biology of Retinoic Acid Receptors. FASEB J. 10, 940-954.

(2) Di Masi, A., Leboffe, L., De Marinis, E., Pagano, F., Cicconi, L., Rochette-Egly, C., Lo-Coco, F., Ascenzi, P., and Nervi, C. (2015) Retinoic Acid Receptors: From Molecular Mechanisms to Cancer Therapy. Mol. Aspects Med. 41, 1-115.

(3) Altucci, L., Leibowitz, M. D., Ogilvie, K. M., De Lera, Á. R., and Gronemeyer, H. (2007) RAR and RXR Modulation in Cancer and Metabolic Disease. Nat. Rev. Drug Discovery 6, 793-810.

(4) Renaud, J. P., Rochel, N., Ruff, M., Vivat, V., Chambon, P., Gronemeyer, H., and Moras, D. (1995) Crystal Structure of the RAR $\gamma$ Ligand-Binding Domain Bound to All-Trans Retinoic Acid. Nature 378, 681-689.

(5) Bourguet, W., Ruff, M., Chambon, P., Gronemeyer, H., and Moras, D. (1995) Crystal Structure of the Ligand-Binding Domain of the Human Nuclear Receptor RXR- $\alpha$. Nature 375, 377-382.

(6) Rastinejad, F., Wagner, T., Zhao, Q., and Khorasanizadeh, S. (2000) Structure of the RXR-RAR DNA-Binding Complex on the Retinoic Acid Response Element DR1. EMBO J. 19, 1045-1054.

(7) Bourguet, W., Vivat, V., Wurtz, J., Chambon, P., Gronemeyer, H., and Moras, D. (2000) Crystal Structure of a Heterodimeric Complex of RAR and RXR Ligand-Binding Domains. Mol. Cell 5, 289-298.

(8) Bastien, J., and Rochette-Egly, C. (2004) Nuclear Retinoid Receptors and the Transcription of Retinoid-Target Genes. Gene 328, $1-16$. 
(9) le Maire, A., Álvarez, S., Shankaranarayanan, P., R de Lera, A., Bourguet, W., and Gronemeyer, H. (2012) Retinoid Receptors and Therapeutic Applications of RAR/RXR Modulators. Curr. Top. Med. Chem. 12, 505-527.

(10) Álvarez, R., Vaz, B., Gronemeyer, H., and De Lera, Á. R. (2014) Functions, Therapeutic Applications, and Synthesis of Retinoids and Carotenoids. Chem. Rev. 114, 1-125.

(11) Haffez, H., Khatib, T., McCaffery, P., Przyborski, S., Redfern, C., and Whiting, A. (2018) Neurogenesis in Response to Synthetic Retinoids at Different Temporal Scales. Mol. Neurobiol. 55, 19421950.

(12) Gudas, L. J., and Wagner, J. A. (2011) Retinoids Regulate Stem Cell Differentiation. J. Cell. Physiol. 226, 322-330.

(13) Al Tanoury, Z., Piskunov, A., and Rochette-Egly, C. (2013) Vitamin A and Retinoid Signaling: Genomic and Nongenomic Effects. J. Lipid Res. 54, 1761-1775.

(14) Christie, V. B., Barnard, J. H., Batsanov, A. S., Bridgens, C. E., Cartmell, E. B., Collings, J. C., Maltman, D. J., Redfern, C. P. F., Marder, T. B., Przyborski, S., and Whiting, A. (2008) Synthesis and Evaluation of Synthetic Retinoid Derivatives as Inducers of Stem Cell Differentiation. Org. Biomol. Chem. 6, 3497-3507.

(15) Barnard, J. H., Collings, J. C., Whiting, A., Przyborski, S. A., and Marder, T. B. (2009) Synthetic Retinoids: Structure-Activity Relationships. Chem. - Eur. J. 15, 11430-11442.

(16) Haffez, H., Chisholm, D. R., Valentine, R., Pohl, E., Redfern, C. P. F., and Whiting, A. (2017) The Molecular Basis of the Interactions between Synthetic Retinoic Acid Analogues and the Retinoic Acid Receptors. MedChem Comm 8, 578-592.

(17) Maltman, D. J., Christie, V. B., Collings, J. C., Barnard, J. H., Fenyk, S., Marder, T. B., Whiting, A., and Przyborski, S. A. (2009) Proteomic Profiling of the Stem Cell Response to Retinoic Acid and Synthetic Retinoid Analogues: Identification of Major RetinoidInducible Proteins. Mol. BioSyst. 5, 458-471.

(18) Bauer, J. B., Lippert, W. P., Dörrich, S., Tebbe, D., Burschka, C., Christie, V. B., Tams, D. M., Henderson, A. P., Murray, B. A., Marder, T. B., Przyborski, S. A., and Tacke, R. (2011) Novel SiliconContaining Analogues of the Retinoid Agonist Bexarotene: Syntheses and Biological Effects on Human Pluripotent Stem Cells. ChemMedChem 6, 1509-1517.

(19) Gentile, M., Latonen, L., and Laiho, M. (2003) Cell Cycle Arrest and Apoptosis Provoked by UV Radiation-Induced DNA Damage Are Transcriptionally Highly Divergent Responses. Nucleic Acids Res. 31, 4779-4790.

(20) Vivas, M. G., Siqueira, J. P., Silva, D. L., de Boni, L., and Mendonca, C. R. (2015) Investigation of the Nonlinear Absorption Spectrum of All-Trans Retinoic Acid by Using the Steady and Transient Two-Photon Absorption Spectroscopy. RSC Adv. 5, 74531-74538.

(21) Presiado, I., Shomer, S., Erez, Y., Gepshtein, R., Amdursky, N., and Huppert, D. (2013) Time-Resolved Emission of Retinoic Acid. J. Photochem. Photobiol., A 258, 30-40.

(22) Murayama, A., Suzuki, T., and Matsui, M. (1997) Photoisomerization of Retinoic Acids in Ethanol under Room Light: A Warning for Cell Biological Study of Geometrical Isomers of Retinoids. J. Nutr. Sci. Vitaminol. 43, 167-176.

(23) Dehu, C., Meyers, F., and Bredas, J. L. (1993) Donor-Acceptor Diphenylacetylenes: Geometric Structure, Electronic Structure, and Second-Order Nonlinear Optical Properties. J. Am. Chem. Soc. 115, 6198-6206.

(24) Nguyen, P., Lesley, G., Marder, T. B., Ledoux, I., and Zyss, J. (1997) Second-Order Nonlinear Optical Properties of Push - Pull Bis(Phenylethynyl)Benzenes and Unsymmetric Platinum Bis(Phenylacetylide) Complexes. Chem. Mater. 9, 406-408.

(25) Hirata, Y., Okada, T., and Nomoto, T. (1997) Photoinduced Intramolecular Charge Separation of P-N, N-Dimethylamino-p ' -Cyano-Diphenylacetylene in Polar Solvents. Chem. Phys. Lett. 278, 133-138.

(26) Fasina, T. M., Collings, J. C., Burke, J. M., Batsanov, A. S., Ward, R. M., Albesa-Jové, D., Porrès, L., Beeby, A., Howard, J. A. K.,
Scott, A. J., Clegg, W., Watt, S. W., Viney, C., and Marder, T. B. (2005) Synthesis, Optical Properties, Crystal Structures and Phase Behaviour of Symmetric, Conjugated Ethynylarene-Based Rigid Rods with Terminal Carboxylate Groups. J. Mater. Chem. 15, 690-697.

(27) Collings, J. C., Parsons, A. C., Porrès, L., Beeby, A., Batsanov, A. S., Howard, J. A. K., Lydon, D. P., Low, P. J., Fairlamb, I. J. S., and Marder, T. B. (2005) Optical Properties of Donor-Acceptor Phenylene-Ethynylene Systems Containing the 6-Methylpyran-2One Group as an Acceptor. Chem. Commun., 2666-2668.

(28) Clemens, G., Flower, K. R., Gardner, P., Henderson, A. P., Knowles, J. P., Marder, T. B., Whiting, A., and Przyborski, S. (2013) Design and Biological Evaluation of Synthetic Retinoids: Probing Length vs. Stability vs. Activity. Mol. BioSyst. 9, 3124-3134.

(29) Nguyen, P., Yuan, Z., Agocs, L., Lesley, G., and Marder, T. B. (1994) Synthesis of Symmetric and Unsymmetric 1,4-Bis(p-RPhenylethynyl)Benzenes via Palladium/Copper Catalyzed CrossCoupling and Comments on the Coupling of Aryl Halides with Terminal Alkynes. Inorg. Chim. Acta 220, 289-296.

(30) Mohan, P., and Rapoport, N. (2010) Doxorubicin as a Molecular Nanotheranostic Agent: Effect of Doxorubicin Encapsulation in Micelles or Nanoemulsions on the Ultrasound-Mediated Intracellular Delivery and Nuclear Trafficking. Mol. Pharmaceutics 7, 1959-1973.

(31) Chisholm, D. R., Zhou, G.-L., Pohl, E., Valentine, R., and Whiting, A. (2016) Practical Synthetic Strategies towards Lipophilic 6-Iodotetrahydroquinolines and -Dihydroquinolines. Beilstein J. Org. Chem. 12, 1851-1862.

(32) Barnard, J. H. (2010) Synthetic Retinoids, Durham University, Durham, U.K.

(33) GB 1417957.6, 2014.

(34) Gluyas, J. B. G., Burschka, C., Dörrich, S., Vallet, J., Gronemeyer, H., and Tacke, R. (2012) Disila-Analogues of the Synthetic Retinoids EC23 and TTNN: Synthesis, Structure and Biological Evaluation. Org. Biomol. Chem. 10 (34), 6914-6929.

(35) Beard, R. L., Teng, M., Colon, D. F., Duong, T. T., Thacher, S. M., Arefieg, T., and Chandraratna, R. A. S. (1997) Synthesis and Biological Activity of 1,2,3,4-Tetrahydroquinoline and 3,4-(1H)Dihydroquinolin-2-One Analogs of Retinoic Acid. Bioorg. Med. Chem. Lett. 7, 2373-2378.

(36) Chisholm, D. R., Lamb, R., Pallett, T., Affleck, V., Holden, C., Marrison, J., O’Toole, P., Ashton, P. D., Newling, K., Steffen, A., Nelson, A. K., Mahler, C., Valentine, R., Blacker, T. S., Bain, A. J., Girkin, J. M., Marder, T. B., Whiting, A., and Ambler, C. A. Photoactivated Cell-Killing Involving a Low Molecular Weight, Donor-Acceptor Diphenylacetylene. Manuscript submitted to Chemical Science.

(37) Redfern, C. P., and Wilson, K. E. (1993) Ligand Binding Properties of Human Cellular Retinoic Acid Binding Protein II Expressed in E. Coli as a Glutathione-S-Transferase Fusion Protein. FEBS Lett. 321, 163-168.

(38) Majumdar, A., Petrescu, A. D., Xiong, Y., and Noy, N. (2011) Nuclear Translocation of Cellular Retinoic Acid-Binding Protein II Is Regulated by Retinoic Acid-Controlled SUMOylation. J. Biol. Chem. 286, 42749-42757.

(39) Sessler, R. J., and Noy, N. (2005) A Ligand-Activated Nuclear Localization Signal in Cellular Retinoic Acid Binding Protein-II. Mol. Cell 18, 343-353.

(40) Vasileiou, C., Lee, K. S. S., Crist, R. M., Vaezeslami, S., Goins, S. M., Geiger, J. H., and Borhan, B. (2009) Dissection of the Critical Binding Determinants of Cellular Retinoic Acid Binding Protein II by Mutagenesis and Fluorescence Binding Assay. Proteins: Struct., Funct., Genet. 76, 281-290.

(41) Kleywegt, G. J., Bergfors, T., Senn, H., Le Motte, P., Gsell, B., Shud, K., and Jones, T. A. (1994) Crystal Structures of Cellular Retinoic Acid Binding Proteins I and II in Complex with All-TransRetinoic Acid and a Synthetic Retinoid. Structure 2, 1241-1258.

(42) Goutelle, S., Maurin, M., Rougier, F., Barbaut, X., Bourguignon, L., Ducher, M., and Maire, P. (2008) The Hill Equation: A Review of 
Its Capabilities in Pharmacological Modelling. Fundam. Clin. Pharmacol. 22, 633-648.

(43) Vaezeslami, S., Mathes, E., Vasileiou, C., Borhan, B., and Geiger, J. H. (2006) The Structure of Apo-Wild-Type Cellular Retinoic Acid Binding Protein II at $1.4 \AA$ and Its Relationship to Ligand Binding and Nuclear Translocation. J. Mol. Biol. 363, 687701.

(44) Pettersen, E. F., Goddard, T. D., Huang, C. C., Couch, G. S., Greenblatt, D. M., Meng, E. C., and Ferrin, T. E. (2004) UCSF Chimera-a Visualization System for Exploratory Research and Analysis. J. Comput. Chem. 25, 1605-1612.

(45) Haffez, H., Chisholm, D. R., Tatum, N. J., Valentine, R., Redfern, C., Pohl, E., Whiting, A., and Przyborski, S. (2018) Probing Biological Activity through Structural Modelling of Ligand-Receptor Interactions of 2,4-Disubstituted Thiazole Retinoids. Bioorg. Med. Chem. 26, 1560-1572.

(46) Wang, Y., Bell, J. C., Keeney, D. S., and Strobel, H. W. (2010) Gene Regulation of CYP4F11 in Human Keratinocyte HaCaT Cells. Drug Metab. Dispos. 38, 100-107. 